\title{
Dynamics, Optimization and Control of a Fuel Cell Based Combined Heat Power (CHP) System for Shipboard Applications
}

\author{
Vasilis Tsourapas, Anna Stefanopoulou and Jing Sun
}

\begin{abstract}
In this paper, a natural gas fuel processor system (FPS), a proton exchange membrane fuel cell (PEM-FC) and a catalytic burner $(\mathrm{CB})$ are integrated in a combined heat power (CHP) generation plant. The $\mathrm{FC}$ provides the power based on electrochemical reaction of hydrogen $\left(\mathrm{H}_{2}\right)$. The FPS generates the hydrogen from natural gas and the $\mathrm{CB}$ provides the energy for preheating the FPS inlet flows by burning any excess $\mathrm{H}_{2}$ from the $\mathrm{FC}$ exhaust. The coupling of these three systems poses a challenging optimization and control problem. The goal is to analyze the open loop dynamics and design a controller that achieves optimal steady state operation and acceptable transient performance, i.e., mitigates the $\mathrm{H}_{2}$ starvation and regulates reactor temperatures. We show in simulations that an observer based feedback controller, which relies on temperature measurements of two reactors, speeds up the transient response fivefold, as compared to the baseline when no feedback control is employed.
\end{abstract}

\section{INTRODUCTION AND MOTIVATION}

Combined heat power (CHP) systems based on fuel cells and fuel processing technologies have great potential for future shipboard applications. In addition to the benefits offered by implementing fuel cell and fuel processor technology within the all-electric ship framework, such as cleaner shipboard power generation, efficient electric power distribution, flexible hull design, silent operation and low thermal acoustic signature, higher efficiency can be achieved by the CHP systems with the incorporation of a catalytic burner. For steady state operations, as much as $16 \%$ efficiency improvement can be achieved with the CHP systems over the stand alone fuel cell power generation systems [1].

The CHP system, depicted in Fig. 1, consists of a proton exchange membrane fuel cell (PEM-FC) stack that generates electric power, a fuel processing system (FPS) that converts a hydrocarbon fuel to a $\mathrm{H}_{2}$ rich mixture to be fed to the fuel cells, and a CB that recuperates any excessive $\mathrm{H}_{2}$ leaving the fuel cells to provide the preheating energy for the reformer. Other auxiliaries, such as the air blower, fuel pump, heat exchangers, are also integral parts of the CHP system. The complicated configuration and

This work is funded in part by the National Science Foundation under contract NSF-CMS-0201332 and the Automotive Research Center (ARC) under U.S. Army contract DAAE07- 98-3-0022, and in part by the Department of Defense under U.S. Navy contract N00014-05-1-0044

V. Tsourapas and J. Sun are with the Department of Naval Architecture \& Marine Engineering, University of Michigan, NAME Bldg, 2600 Draper Rd. Ann Arbor, MI 48109-2145 djvaseumich. edu, jingsun@umich.edu

A. Stefanopoulou is with the Department of Mechanical Engineering, University of Michigan, 2043 WE Lay Auto Lab, 1231 Beal Ave. Ann Arbor MI 48109-2121 annastef@umich.edu closely coupled dynamics, together with the large thermal inertia and therefore slow transients associated with the fuel processor, impose a very challenging control problem.

In order to achieve the overall maximum efficiency, the integrated CHP system has to work very close to its operating boundary (Fig. 3). This optimal set-point selection, however, puts the system in a very vulnerable position during system transitions. When a load is suddenly applied, the CHP system may not be able to provide sufficient $\mathrm{H}_{2}$ and heat fast enough to sustain the operation under the new load condition, thereby leading to temporarily $\mathrm{H}_{2}$ starvation or system shutdown. These problems can be avoided by slowing down the load drawn from the fuel cell through a slew rate or a load governor [2]. The load deficit in this case can be provided through hybridization [3], with an additional electrical power source which can increase cost and complexity. Another typical approach that does not rely on hybridization is to increase the system operating range by producing high rates of hydrogen, thus lowering FC utilization and CHP efficiency.

Operating at optimal steady state and thus capitalizing the benefits of the CHP system will be made possible only if the controlled system responds very fast to FC load changes. Otherwise, one has to resort to sub-optimal setpoints, i.e., to trade the efficiency for improved safety margin. In this paper we design a controller that speedsup the natural CHP dynamics and supplies hydrogen to the fuel cell while maintaining optimum reactor temperatures. The feedback controller is based on measuring reactor temperatures and estimating the spatially averaged composition of reactant flow through the series of $\mathrm{CHP}$ components.

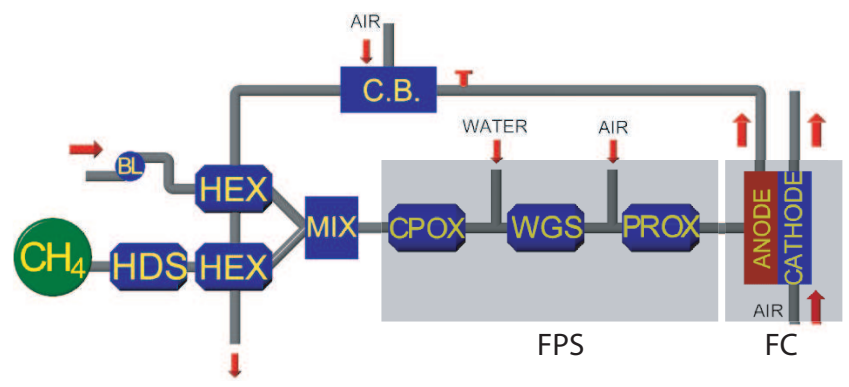

Fig. 1. A CHP system consisting of FPS, FC and CB.

\section{AN OVERVIEW OF THE CHP SYSTEM}

A low pressure CHP system with a rated power of $250 \mathrm{~kW}$ is used as the platform for our investigation in this 
paper. For shipboard power applications, a key enabling technology is the on-board fuel reforming, and a critical requirement is the fast load following capability. In this application, we consider the catalytic partial oxidation reforming technology, because of its fast (relative to steam reforming) transient response.

The FPS, depicted in Fig. 1, is composed of four main reactors, namely, the hydro-desulfurizer (HDS), the catalytic partial oxidation (CPOX), the water gas shift (WGS), and the preferential oxidation (PROX). Natural gas, rich in methane $\mathrm{CH}_{4}$, is supplied to the FPS from a tank. All FPS and FC components operate at low pressures of up to 110 $\mathrm{kPa}$. The HDS is used to remove the sulfur from the natural gas [4], [5]. The main air flow is supplied to the system by a blower (BL) which draws air from the atmosphere. The air and the de-sulfurized fuel are pre-heated in separate heat exchangers (HEX), before they are mixed in the mixer (MIX). The mixture is then passed through the catalytic partial oxidizer (CPOX) where $\mathrm{CH}_{4}$ reacts with oxygen to produce $\mathrm{H}_{2}$. There are two main chemical reactions taking place in the CPOX: partial oxidation (POX) and total oxidation (TOX) [6], [7]:

$$
\begin{array}{cc}
(\mathrm{POX}) & \mathrm{CH}_{4}+\frac{1}{2} \mathrm{O}_{2} \rightarrow \mathrm{CO}+2 \mathrm{H}_{2} \\
\Delta H_{\text {pox }}^{0}=-0.036 \times 10^{6} \mathrm{~J} / \mathrm{mol} \\
(\mathrm{TOX}) \quad \mathrm{CH}_{4}+2 \mathrm{O}_{2} \rightarrow \mathrm{CO}_{2}+2 \mathrm{H}_{2} \mathrm{O} \\
\Delta H_{\text {tox }}^{0}=-0.8026 \times 10^{6} \mathrm{~J} / \mathrm{mol} .
\end{array}
$$

Hydrogen is produced only by the POX reaction while heat is mostly generated by the TOX reaction. As shown in Fig. 2, the balance between the two is dictated by the molar ratio of $\mathrm{O}_{2}$ to $\mathrm{CH}_{4}, \lambda_{O 2 C}=n_{\mathrm{O}_{2}} / n_{C_{4}}$, where $n_{i}$ is the number of moles of the species $i$. Moreover, since the CPOX products are also highly dependent on the CPOX reactor temperature $T_{C P O X}$, the optimum balance between the two reactions has to be determined.

Carbon monoxide (CO) is also created along with $\mathrm{H}_{2}$ in the POX reaction, as can be seen in (1). Since CO poisons the PEM fuel cell catalyst, it has to be eliminated using water in the gas shift converter (WGS) and air in the preferential oxidizer (PROX). The later are assumed to operate perfectly thus eliminating all the $\mathrm{CO}$ in the stream. The $\mathrm{H}_{2}$-rich mixture leaving the PROX enters the anode of the fuel cell stack where the electro-chemical reaction
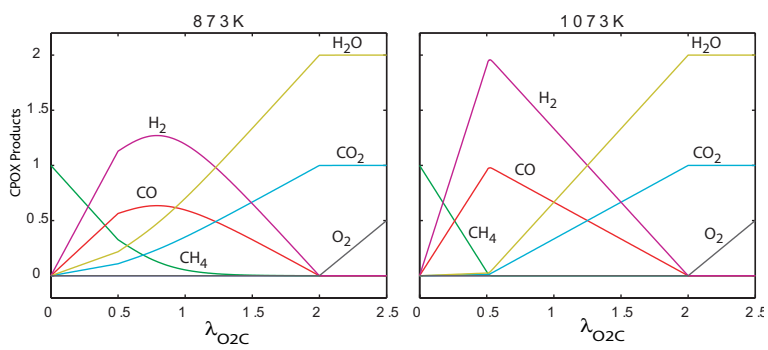

Fig. 2. CPOX products as a function of $\lambda_{O 2 C}$ and reactor temperature takes place to convert $\mathrm{H}_{2}$ to electrical power. The flow from the anode is then supplied to the catalytic burner (CB) where the excess $\mathrm{H}_{2}$ is burnt using the air supplied through a blower. Finally, the flow from the $\mathrm{CB}$ is fed to two separate heat exchangers (HEX), one to preheat the air and one to pre-heat the fuel before they enter the FPS and thereby increasing the overall utilization of the fuel.

In [1], a 19 state, nonlinear, dynamic and control oriented model for the CHP system has been developed based on the fuel processor system in [8]. This model is employed in this work for optimization analysis, performance evaluation and control design.

\section{Steady State EFFiCiEnCy Optimization}

For the system to work efficiently in an integrated fashion, each component has to be conditioned properly in terms of its operating temperature, humidity, and pressure. This is achieved by controlling the air and fuel intakes of the FPS. The strong physical coupling of the CHP components, shown in Fig. 1, will eventually dictate the optimal set-points for the system.

To determine the optimal steady state operating points with respect to the overall system efficiency, the following optimization problem is formulated

$$
\max _{\left(u_{f}, u_{a}\right)}\left(\eta_{C H P}=\frac{V \cdot I_{s t}}{W_{u s e d}^{C H_{4}} \cdot Q_{L H V}^{C H_{4}}}\right) .
$$

The objective is to maximize the overall efficiency, $\eta_{C H P}$, which is defined as the ratio of the FC output power $V \cdot I_{s t}$ over the energy used $W_{u s e d}^{C_{4} H_{4}} \cdot Q_{L H V}^{C H_{4}}$, where $W_{u s e d}^{C H_{4}}$ is the amount of fuel used and $Q_{\mathrm{LHV}}^{\mathrm{CH}_{4}}$ its lower heating value. The optimization variables are the fuel valve command, $u_{f}$, and the air blower command, $u_{a}$, both ranging from 0 to $100 \%$, corresponding to minimum and maximum actuator inputs respectively, or to minimum and maximum flows.

The modified gradient descent method was employed to solve the optimization problem [9]. The corresponding iterative algorithm is given as

$$
u_{k+1}=u_{k}-a_{k} \cdot \nabla \eta_{C H P}^{T}\left(x_{k}\right)
$$

where $a_{k}$ is the iteration step, $\nabla \eta_{C H P}^{T}\left(x_{k}\right)$ is the gradient vector which corresponds to the $\eta_{C H P}$ increasing direction and $u=\left[\begin{array}{ll}u_{f} & u_{a}\end{array}\right]^{T}$. The iteration step size, $a_{k}$, is kept constant until no further increasing steps can be found and is then reduced by the bisection method up to the desired accuracy.

Convergence of the gradient algorithm to a global maximum can be verified given the convex form of the efficiency map for the whole range of FC loads. An example of the efficiency map is given in Fig. 3 for the FC load ${ }^{1}$ of $100 \mathrm{~A}$. The maximum efficiency for this load is $33.6 \%$ while the optimal setpoint is $\left[\begin{array}{ll}u_{f}^{*} & u_{a}^{*}\end{array}\right]=\left[\begin{array}{ll}20.75 & 29.00\end{array}\right]$. Using the same procedure for each operating load the

\footnotetext{
${ }^{1}$ In this paper, the term "load" is synonymous to the current drawn from the fuel cell.
} 


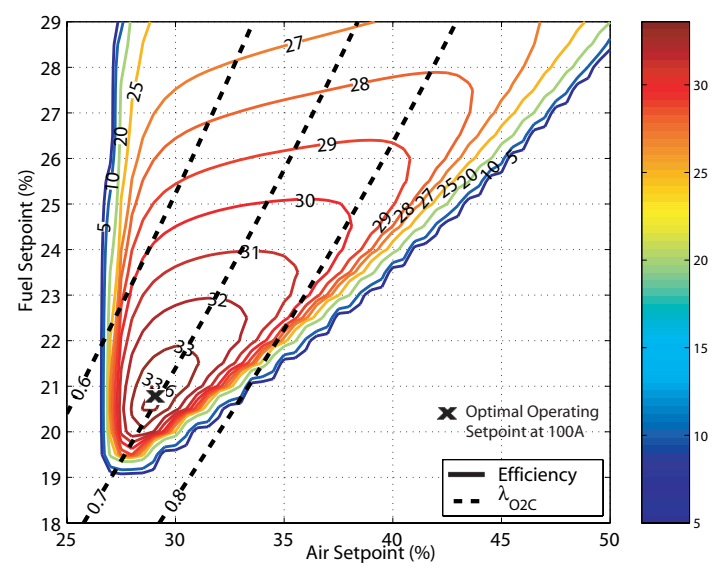

Fig. 3. Efficiency map of the CHP system at load=100A

optimal steady state setpoint map can be determined for the actuator inputs $u^{*}=\left[\begin{array}{ll}u_{f}^{*} & u_{a}^{*}\end{array}\right]^{T}=f_{u}\left(I_{s t}\right)$ and other critical operating variables (eg. $T_{\text {cpox }}^{*}=f_{\text {cpox }}\left(I_{s t}\right)$ ) that can serve later as controller setpoints.

As illustrated in Fig. 3, the optimal operating setpoint lies close to the operating boundary of the system. This trend is observed for the whole range of operating loads of the system. The operating boundary $\left(\eta_{C H P}=0 \%\right)$ is defined as the locus of points where the produced amount of $\mathrm{H}_{2}$ is less than the required amount by the $\mathrm{FC}$, given a specific load at steady state operation. As a result, the system is susceptible to steady state $\mathrm{H}_{2}$ starvation during uncompensated loads. To avoid modeling errors and react fast to load variations, a combination of feedforward and feedback control is required.

It is important to point out, that according to the optimization results, all the optimal operating points, independent of the load drawn from the FC, occur at $\lambda_{O 2 C}^{*}=0.69$ and $T_{C P O X}^{*}=980 \mathrm{~K}$. Note here that the optimization of the overall CHP efficiency (3), corresponds to a CPOX oxygen to carbon ratio $\left(\lambda_{O 2 C}^{*}\right)$ that is greater than the value for maximum $\mathrm{H}_{2}$ production at the CPOX reactor $\left(\lambda_{O 2 C}=0.5\right.$ as indicated in Fig. 2)

In order to explain this result, one has to notice that while both POX and TOX reactions in (1)-(2) are exothermal, the TOX releases 20 times more heat than the POX reaction. For the integrated CHP system the CPOX temperature, which is highly coupled to the $\mathrm{H}_{2}$ production, is a function of both the heat released by the reactions inside the CPOX and the temperature of the incoming air and fuel flows which are heated by the energy supplied through the CB. Moving $\lambda_{O 2 C}$ towards 0.5 , will promote $\mathrm{H}_{2}$ production but suppress the TOX reaction which only occurs for $\lambda_{O 2 C}>0.5$. Thus the contribution of the TOX reaction to the CPOX temperature will be reduced and, as a result, the CPOX reactor has to rely on preheating the inlet flows by the CB. Since both outputs (the $\mathrm{H}_{2}$ and heat) are essential for the system to function properly, $\lambda_{O 2 C}^{*}=$ 0.69 , the optimal point, reflects a balance between the $\mathrm{H}_{2}$ production in the FPS and heat generation externally in the $\mathrm{CB}$ and internally in the CPOX reactors for steady state operation. It is important to note that the overall CHP optimum is not defined through optimization of individual components.

\section{OPEN LOOP DYNAMICS}

Examining the transient performance of the system using static feedforward control enables us to gain insight on the system dynamics. In this section we examine the open loop system dynamics by utilizing the optimal steady state setpoint maps derived from the optimization results described in Sec. III. For a given load, the fuel and air operating setpoint are defined by the feedforward maps. Since the maps were optimized for steady state operation the open loop control is not adequate to prevent $\mathrm{H}_{2}$ starvation when a large step change in load is demanded.

The FPS introduces a considerable lag in $\mathrm{H}_{2}$ generation due to the multiple volumes and reactors involved, thus has a slower time constant than the fuel cell stack when a step change in the demanded load is applied. Consequently, the maximum possible rate at which the load can be increased is dictated by the FPS and the $\mathrm{H}_{2}$ production dynamics.

The open loop response of the system for two consecutive load steps, one 90-100A and another 100-150A, is shown in Fig. 4. For the initial small step of 90-100A, the fuel processor manages to provide the fuel cell with the required amount of $\mathrm{H}_{2}$ in order to meet the load demand. For the second larger step of 100-150A though, the $\mathrm{H}_{2}$ generation is below the demanded $\mathrm{H}_{2}$ level for a considerable period of 10 seconds. Starving a fuel cell for 10 seconds can cause extended membrane damage and jeopardizes the life span of the stack. Moreover, a
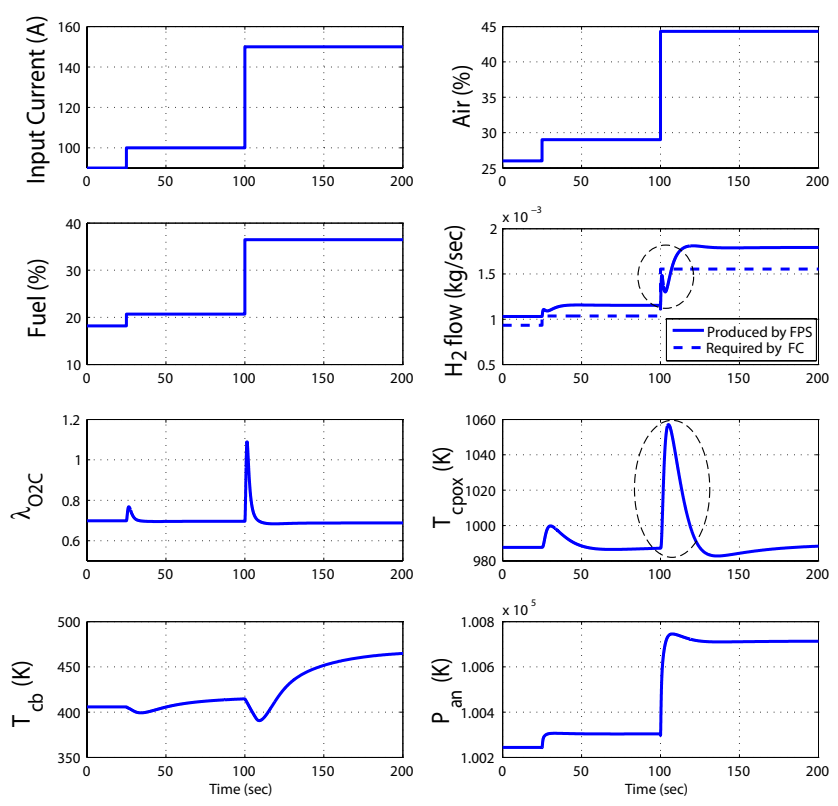

Fig. 4. Open Loop Performance during 90-100A and 100-150A steps 

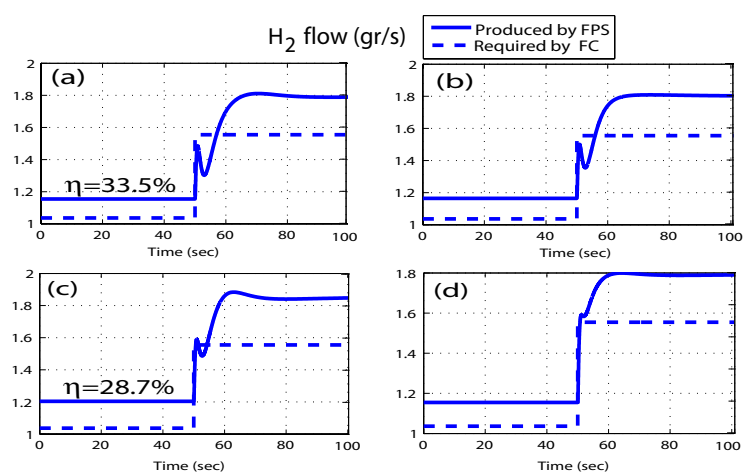

Fig. 5. Transient Performance Comparison of (a) original system (b) system with constant preheating temperature (c) system with suboptimal maps (d) system with HDS volume of $0.1 \mathrm{~m}^{3}$

$65 \mathrm{~K}$ overshoot in the CPOX temperature within $15 \mathrm{sec}$ is observed, which can have damaging consequences for the CPOX reactor. Both issues are highlighted on Fig. 4.

There are three critical processes that affect the generation of $\mathrm{H}_{2}$ during load changes and cause the $\mathrm{H}_{2}$ starvation problem. Analyzing those processes will provide insight in the control problem and the system design.

The first critical process was found to be the $\mathrm{CB}$ temperature variation during a step change in load. When a step load is applied the $\mathrm{H}_{2}$ flow is depleted at a rate faster than it is produced, due to the slow time constant of the FPS. This results in reduction or even elimination of the $\mathrm{H}_{2}$ flow to the catalytic burner, which in turn results in a temperature reduction in the $\mathrm{CB}$ and eventually a temperature reduction of the inlet air and fuel flows. However, the thermal inertia and large time constant of the $\mathrm{CB}$ can maintain the temperature to a level that does not affect the $\mathrm{H}_{2}$ production of the FPS. A comparison of the CHP system, where the $\mathrm{CB}$ temperature is a function of $\mathrm{H}_{2}$ in anode exhaust with an imaginary system where the $\mathrm{CB}$ temperature is maintained constant at a nominal value is given in Fig. 5a and b. The two responses are almost identical, with the constant $\mathrm{CB}$ temperature response (Fig. 5b) exhibiting slightly less undershoot. Consequently, the $\mathrm{CB}$ temperature variation during load increase is not the main cause of the $\mathrm{H}_{2}$ starvation problem.

As mentioned earlier the feedforward controller based on the steady state optimization places the system close to its operating boundaries and therefore making it susceptible to $\mathrm{H}_{2}$ starvation. A non-optimal map implies increased excess fuel usage which implies increased $\mathrm{H}_{2}$ production in steady state. As a result the difference between the required $\mathrm{H}_{2}$ and the produced is expanded leading to increased safety margins at the price of reduced efficiency. In the case shown in Fig. 5c, there is an efficiency reduction from $33.5 \%$ to $28.7 \%$.

The third and most important cause of the $\mathrm{H}_{2}$ starvation can be attributed to reactor sizing issues. The large residence time of the HDS, due to the slow kinetics of
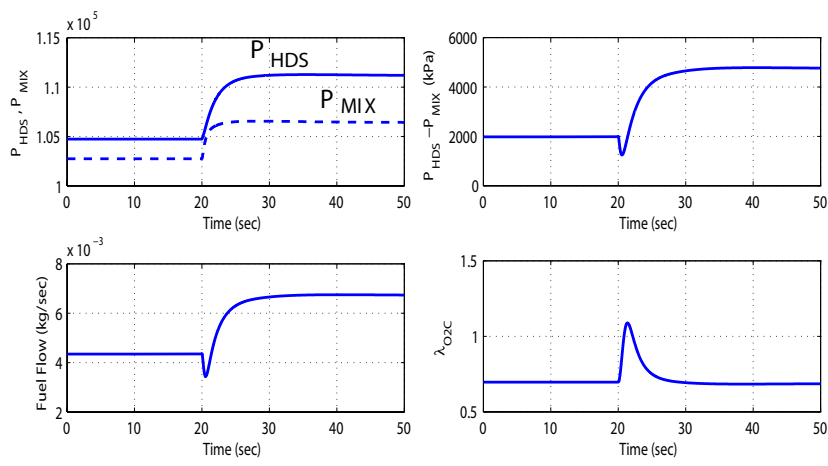

Fig. 6. $\lambda_{O 2 C}$ overshoot during open loop operation

the fuel desulphurization [10], imposes the requirement of a relatively large volume compared to the adjacent volumes of the MIX and the HEX. The combination of the large volume in the fuel path, the small volume of the air path and the large flow of air compared to the fuel flow causes the MIX pressure to built up at a rate faster than the HDS pressure during transients. In turn the pressure difference between those volumes initially exhibits an undershoot until the HDS pressure manages to built up again as illustrated in Fig. 6. Since the fuel flow is a function of the pressure gradient, the same undershoot is observed for the fuel flow which causes the oxygen to carbon ratio $\lambda_{O 2 C}$, to overshoot.

Based on the CPOX reaction map, given in Fig. 2, an overshoot in $\lambda_{O 2 C}$ from its nominal steady state operating point 0.69 to 1.15 implies a steep decrease in $\mathrm{H}_{2}$ production. One way to illustrate this is by decreasing the HDS volume in the model. Fig. 5d compares the response of the original system, with $V_{H D S}=0.3 \mathrm{~m}^{3}$, with an imaginary system that has a considerably smaller HDS volume of $0.1 \mathrm{~m}^{3}$. For the later case, the $\mathrm{H}_{2}$ starvation problem disappeared. If future advances in the desulphurization process produce a compact HDS reactor, then the transient performance of the system would improve dramatically.

\section{Feedback Control Design \& Analysis}

In this section we investigate the effectiveness of using observer based feedback control in improving the transient performance of the CHP system. In particular, our objective is to eliminate the $\mathrm{H}_{2}$ starvation problem and to control the CPOX temperature overshoot while maintaining efficient steady state operation by utilizing the optimized feedforward maps.

\section{A. Selection of Feedback Variables}

The control architecture is based on setpoint error regulation, using the setpoint maps defined in Sec. III through the plant optimization. The controller is implemented by augmenting integrators to the estimator based feedback controller. In deciding which signals need to be regulated and are best suited as feedback variables in the controller, 
one has to consider the control requirements (namely, $\mathrm{H}_{2}$ and $T_{\text {cpox }}$ regulation), the sensitivity of measured signals to the fuel and air actuators, as well as the ease of measuring those signals. Ideally we would choose the CPOX temperature $T_{\text {cpox }}$ and the partial pressure of $\mathrm{H}_{2}$ leaving the anode $P_{\mathrm{H}_{2}}$ as the feedback variables, as they are linked directly to the control objectives. However, since $P_{\mathrm{H}_{2}}$ is difficult to measure, we choose the $\mathrm{CB}$ temperature $T_{c b}$ instead, which is closely coupled to the the $\mathrm{H}_{2}$ starvation problem. During transient operation, reduction of $T_{c b}$ from its optimal steady state value $T_{c b}^{*}$ implies reduction of the $\mathrm{H}_{2}$ leaving the anode exhaust or in turn $\mathrm{H}_{2}$ starvation. The only drawback of using $T_{c b}$ instead of $P_{H_{2}}$ is the slow dynamics due to the associated thermal inertia.

It should be also pointed out that the sensitivity plots, shown in Fig. 7, illustrate that for the optimal setpoint $\lambda_{O 2 C}=0.69$, the $\mathrm{CB}$ temperature reaches the peak value as air is varied for a certain FC load (100A). Linearization at this operating point results in zero DC gain between the air command and the $T_{c b}$. As a result we expect the fuel command to define the steady state $T_{c b}$ and the air command to define steady state $T_{\text {cpox }}$. The emerging inputoutput coupling at the steady state clearly suggests that air should be used to control $T_{c p o x}$ and the fuel to control $T_{c b}$ in the case of decentralized controller design.

\section{B. Linearization}

The nonlinear CHP model developed in [1] can be expressed as

$$
\dot{x}=f(x, u, w) .
$$

The model has 19 states and two inputs, namely the fuel and air command. The current drawn from the FC stack is treated as a disturbance to the system and finally the feedback variables are $T_{c p o x}$ and $T_{c b}$.

$$
u=\left[\begin{array}{ll}
u_{f} & u_{a}
\end{array}\right]^{T}, \quad w=I_{s t}, \quad z=\left[\begin{array}{ll}
T_{\text {cpox }} T_{c b}
\end{array}\right]^{T} .
$$

For control design, a linear expression of the CHP plant is obtained using MATLAB/Simulink(C), where the model of the system has also been developed. The medium load of $100 \mathrm{~A}(115 \mathrm{~kW})$ was chosen as the linearization point. An expression of the linear plant is given by

$\delta \dot{x}=A_{p} \cdot \delta x+B_{p} \cdot \delta u+B_{w} \cdot \delta w \quad, \quad \delta z=C_{z} \cdot \delta x$.

where $\delta(\cdot)=(\cdot)-(\cdot)_{0}$ and $(\cdot)_{0}$ is the nominal operating point at the linearization load.
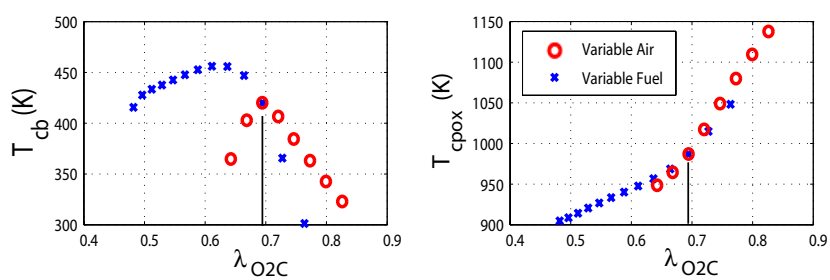

Fig. 7. Steady State Operating Points for variable Air and Fuel Inputs

\section{Feedback Control Design}

A full state feedback controller is designed using the LQR technique. For the tracking problem we augment the plant states with the integrator states:

$$
\delta \dot{q}=\left[\begin{array}{c}
T_{\text {cpox }}-T_{c p o x}^{*} \\
T_{c b}-T_{c b}^{*}
\end{array}\right]
$$

where $T_{c p o x}^{*}$ and $T_{c b}^{*}$ are the corresponding desired temperatures, which depend on the FC operating load and can be computed off-line from the optimization procedure and stored for on-line implementation.

The control signal can be expressed as $\delta u=$ $-\left[\begin{array}{ll}K_{P} & K_{I}\end{array}\right] \cdot \delta z_{\text {aug }}$, where $\delta z_{\text {aug }}=\left[\begin{array}{ll}\delta x & \delta q\end{array}\right]^{T}, K_{P} \epsilon R^{2 x 19}$ is the proportional gain and $K_{I} \epsilon R^{2 x 2}$ is the integral gain. $K_{\text {aug }}=\left[\begin{array}{ll}K_{P} & K_{I}\end{array}\right]=P B_{p}^{T} R_{K}^{-1}$, where $P$ is the solution to the Riccati equation

$$
A_{p} P+P A_{p}^{T}-P B_{p}^{T} R_{K}^{-1} B_{p} P+Q_{K}=0,
$$

is calculated by minimizing the quadratic cost function

$$
J=\int_{0}^{\infty}\left(\delta z_{\text {aug }}^{T} \cdot Q_{K} \cdot \delta z_{\text {aug }}+\delta u^{T} \cdot R_{K} \cdot \delta u\right) d t
$$

The weighting matrices on the controller inputs $Q_{K}$ and the outputs $R_{K}$ are

$$
Q_{K}=\operatorname{diag}\left(\left[\begin{array}{llll}
0.1 & 0.1 & 2 & 1
\end{array}\right]\right), R_{K}=\operatorname{diag}\left(\left[\begin{array}{ll}
1 & 0.1
\end{array}\right]\right) \text {. }
$$

Recognizing that measuring all 19 states is impossible, we design a state estimator to provide the needed information for state feedback. The state estimator is expressed as

$$
\begin{aligned}
& \delta \dot{\hat{x}}=A_{p} \cdot \delta \hat{x}+B_{p} \cdot \delta u+B_{w} \cdot \delta w+L \cdot(\delta z-\delta \hat{z}) \\
& \delta \hat{z}=C_{z} \cdot \delta \hat{x} .
\end{aligned}
$$

where $L=S C_{z}^{T} R_{L}^{-1}$ and $S$ is the solution to

$$
A_{p} S+S A_{p}^{T}-S C_{z}^{T} R_{L}^{-1} C_{z} S+B_{p} Q_{L} B_{p}^{T}=0 .
$$

The observer gain $L$ is designed using the process noise covariance $Q_{L}$ and the measurement noise covariance $R_{L}$ given below

$$
Q_{L}=\operatorname{diag}\left(\left[\begin{array}{ll}
10 & 0.01
\end{array}\right]\right), R_{L}=\operatorname{diag}\left(\left[\begin{array}{ll}
0.01 & 10
\end{array}\right]\right) .
$$

The control signal $u=\left[\begin{array}{ll}u_{f} & u_{a}\end{array}\right]$, when the observer based controller is implemented in the nonlinear CHP model is given by

$$
u=-K_{I} \cdot \delta q-K_{P} \cdot\left(\hat{x}-x_{0}\right)+u_{0}
$$

\section{Vi. Performance Evaluation}

The estimator based feedback control scheme, compared to the open loop controller, manages to improve the transient performance of the CHP system (Fig. 8). It is interesting to note that the feedback controller overshoots fuel and slows down air command initially, in order to regulate $\lambda_{O 2 C}$ around its optimal value and resolve the undershoot of fuel flow that was observed on the open loop system (Fig. 6). As a result, the $\mathrm{H}_{2}$ production increases smoothly and the $\mathrm{H}_{2}$ starvation problem reduced. 

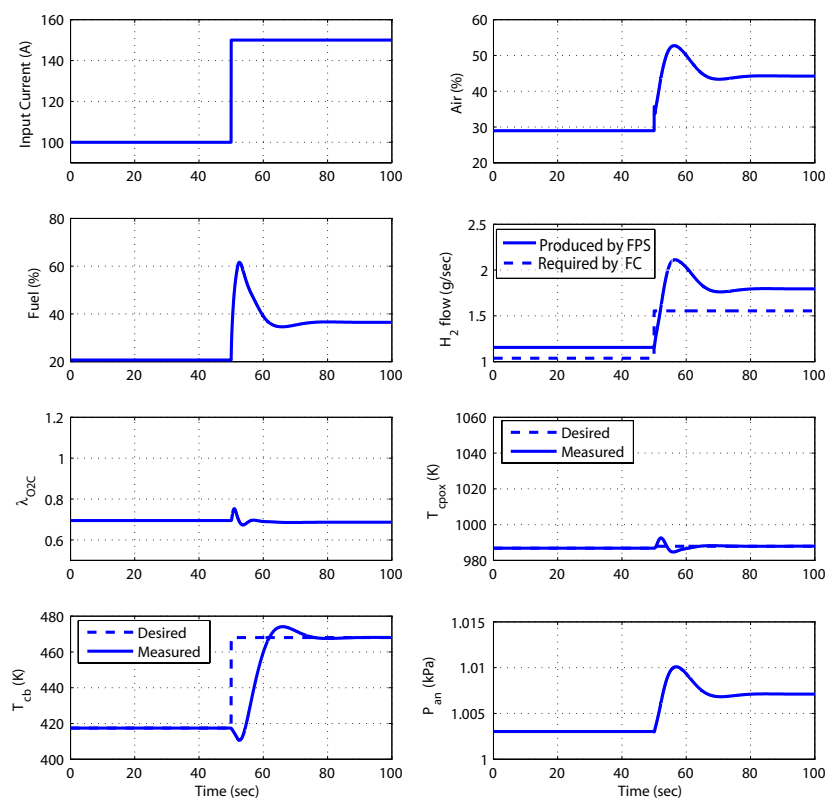

Fig. 8. Response of the closed loop system

Furthermore, the CPOX reactor temperature overshoot is substantially reduced. Application of the observer based feedback controller yields a small overshoot which is negligible compared to the open loop performance where $T_{\text {cpox }}$ overshoots to $1060 \mathrm{~K}$ within $15 \mathrm{sec}$.

Even when feedback control is applied, the CHP transient response still exhibits a short period of $\mathrm{H}_{2}$ starvation which can be attributed to the lag due to the multiple reactor volumes in the flow. A constant rate limiter can be implemented in order to eliminate the starvation by slowing down the rate at which the current is drawn from the FC. Without feedback, a rate limiter of $4 \mathrm{~A} / \mathrm{sec}$ would be required, while for the state or the observer based feedback a rate limiter of $20 \mathrm{~A} / \mathrm{s}$ is adequate to eliminate the problem. Thus the maximum transient speed has been increased by a factor of five.

Finally, it is important to note that the performance achieved with the proposed controller, that utilizes only temperature measurements, is comparable to the performance of the controller developed in [11] for the FPSFC system, that utilizes $T_{\text {cpox }}$ and $y_{H_{2}}=P_{H_{2}}^{a n} / P^{a n}$ (i.e. $\mathrm{H}_{2}$ partial pressure sensor). The later measurement is significantly faster than $T_{c b}$, since it involves pressure dynamics instead of temperature. Thus, implementing a CB into an FPS, besides increasing significantly the steady state efficiency, also provides an indirect measurement of the $\mathrm{H}_{2}$ starvation that is easy to measure and can be utilized to control the transient response. Summarizing the overall $\mathrm{CB}$ contributions to the FPS, we can conclude that the $\mathrm{CB}$

- significantly improves steady state efficiency

- does not degrade the transient performance of the plant due to the thermal inertia

- provides an indirect measurement of the $\mathrm{H}_{2}$ produc- tion via $T_{c b}$ that is otherwise difficult to obtain.

\section{CONCLUSIONS}

In this paper, the control oriented model of a fuel cell based CHP system, developed in [1] is employed for steady state optimization and control design. Using the gradient algorithm, the air and fuel setpoints that yield maximum overall efficiency are determined for every load of the FC. The system characteristics and dynamic properties are analyzed. The processes responsible for the poor transient open loop performance are identified. A feedback control scheme is designed using only the temperature measurements of the CPOX and the CB reactors. Our results show that the $\mathrm{H}_{2}$ starvation is mitigated and the CPOX temperature overshoot is minimized during a large load step when the proposed feedback controller is implemented. Moreover, a $500 \%$ increase in the maximum transient speed of the CHP plant is achieved. Finally it is proven that implementing a $\mathrm{CB}$ into a FC reformer is beneficial to the system. Robustness analysis and complexity reduction of the controller are yet to be examined.

\section{REFERENCES}

[1] V. Tsourapas, J. Sun, and A. Stefanopoulou, "Modeling and dynamics of a fuel cell combined heat power system for marine applications," IASME Transactions, vol. 2, no. 1, pp. 287-293, 2004.

[2] J. Sun and I. Kolmanovsky, "A robust load governor for fuel cell oxygen starvation protection," Proceedings of 2004 American Control Conference, June 2004.

[3] A. Vahidi, A. Stefanopoulou, and H. Peng, "Model predictive control for starvation prevention in a hybrid fuel cell system," Proc. of American Control Conference, no. 834-839, 2004.

[4] A. Dicks, "Hydrogen generation from natural gas for the fuel cell systems of tomorrow," Journal of Power Sources, vol. 61, pp. 113$124,1996$.

[5] T. Gardner, D. Berry, K. Lyons, S. Beer, and A. Freed, "Fuel processor integrated $\mathrm{H}_{2} \mathrm{~S}$ catalytic partial oxidation technology for sulfur removal in fuel cell power plants," Fuel, vol. 81, pp. 21572166, 2002.

[6] A. Larentis, N. de Resende, V. Salim, and J. Pinto, "Modeling and optimization of the combined carbon dioxide reforming and partial oxidation of natural gas," Applied Catalysis, vol. 215, pp. 211-224, 2001.

[7] J. Zhu, D. Zhang, and K. King, "Reforming of $\mathrm{CH}_{4}$ by partial oxidation: thermodynamic and kinetic analyses," Fuel, vol. 80, pp. 899-905, 2001.

[8] J. T. Pukrushpan, A. Stefanopoulou, and H. Peng, Control of Fuel Cell Power Systems : Principles, Modeling, Analysis and Feedback Design. Springer, 1st edition, 2004.

[9] P. Papalambros and D. Wilde, Principles of Optimal Design: Modeling and Computation. Cambridge University Press, 2nd edition, 2000.

[10] K. Tawara and T. Nishimura, "New hydrodesulfurization catalyst for petroleum-fed fuel cell vehicles and cogenerations," Ind. Eng. Chem. Res., 40, 2367-2370, 2001.

[11] J. T. Pukrushpan, A. G. Stefanopoulou, S. Varigonda, L. M. Pedersen, S. Ghosh, and H. Peng, "Control of natural gas catalytic partial oxidation for hydrogen generation in fuel cell applications," IEEE Transactions on Control Systems Technology, vol. 13, no. 1, January 2005 . 